\section{Idiopathische entzündliche Myopathie: Geburtsrisiken untersucht}

Che WI et al. Pregnancy outcomes in women with idiopathic inflammatory myopathy, before and after diagnosis - a population-based study. Rheumatology 2020; 59: 2572-2580

Schwedische Wissenschaftler untersuchten Zusammenhänge von idiopathischer entzündlicher Myopathie (IIM) und unerwünschten Schwangerschaftsergebnissen bei Geburten von Frauen vor und nach der IIM-Diagnose.

Die Forschergruppe griff auf Daten des schwedischen nationalen Patientenregisters (NPR) zu, um Einzelgeburten in den Jahren 1973-2016 bei Frauen zu identifizieren, bei denen zwischen 1998 und 2016 IIM diagnostiziert wurde und bei denen, bei denen dies nicht der Fall war. Die Geburten wurden nach dem IIM-Status der Mutter zum Zeitpunkt der Entbindung klassifiziert: nach IIM ( $n=68), 1-3$ Jahre vor IIM ( $n=23)$, mehr als 3 Jahre vor IIM $(n=710)$ und nicht IIM ausgesetzt ( $n=4101)$. Mit multivariaten Regressionsmodellen wurde das relative Risiko unerwünschter Schwangerschaftsergebnisse bei Geburten nach IIM und vor IIM gegenüber vergleichbaren Frauen ohne IIM getrennt abgeschätzt. Dabei wurden die mütterlichen Merkmale stratifiziert nach Post-IIM- und Pre-IIM-Geburten und mit ihren entsprechenden, in den Merkmalen übereinstimmenden Nicht-IIM-Müttern verglichen.

Während des Studienzeitraums wurden 985 Frauen mit IIM identifiziert. Davon hatten 421 (42,74\%) in der Vergangenheit 801 Einzelgeburten, verglichen mit 2099 (42,99\%) von 4883 Frauen des Gesamtregisters ohne Auftreten einer IIM. Insgesamt hatten alle Frauen der Auswertung 4902 Einzelgeburten (IIM, $\mathrm{n}=801$ und Nicht-IIM, $\mathrm{n}=4101$ ).

Von den 801 Geburten bei Frauen mit IIM kam es in 68 Fällen nach dem Auftreten der IIM zur Geburt und in 736 Fällen vor der IIM. Bei den in den Merkmalen übereinstimmenden Nicht-IIM- Frauen kam es demnach zu 236 bzw. 3865 Geburten.
Post-IIM-Geburten hatten ein signifikant höheres Risiko einer Kaiserschnittgeburt (aRR = 1,98; 95\% KI 1,08-3,64) sowie einer Frühgeburt (aRR=3,35; $95 \%$ KI 1,28-8,73) und ein signifikant höheres Risiko Kinder mit niedrigem Geburtsgewicht zu gebären (aRR = 5,69; 95 \% KI 1,84-17,55). Darüber hinaus waren bei Frühgeburten eine sehr Frühgeburt und eine moderat frühe Frühgeburt häufiger als bei der Vergleichsgruppe ohne IIM.

Keine Unterschiede wurden zwischen PostIIM-Geburten und der Vergleichsgruppe ohne IIM hinsichtlich des Risikos einer instrumentellen Entbindung, einer Geburtseinleitung oder von Infektionen bei den Säuglingen und Kindern gefunden.

Im Vergleich zu den Frauen ohne IIM hatten Frauen, die 1-3 Jahre vor einer IIM-Diagnose gebaren, vermehrt Kaiserschnittgeburten(17,39 vs. $11,17 \%)$ und instrumentelle Entbindungen (17,39 vs. 5,96\%). Die Unterschiede waren nicht signifikant.

Bei Geburten, die mehr als 3 Jahre vor der IIMDiagnose auftraten, waren die Wahrscheinlichkeiten von Kaiserschnitt, Induktion von Wehen, geringem Geburtsgewicht sowie für das Schwangerschaftsalter zu leichte (SGA) Säuglingsgeburten signifikant erhöht.

\section{FAZIT}

Frauen, die nach einer IIM-Diagnose geboren hatten, hatten ein höheres Risiko für Kaiserschnitt, Frühgeburt und niedriges Geburtsgewicht. Nach Auffassung der Autoren unterstreicht dies die Bedeutung einer besonderen Betreuung und einer genauen Überwachung von Frauen mit IIM. Höhere Häufigkeiten des Kaiserschnitts und der instrumentellen Entbindung bei Geburten vor dem Auftreten einer IIM unterstreichen die Notwendigkeit zukünftiger Forschung zum Einfluss subklinischer Merkmale von IIM auf die Schwangerschaftsergebnisse, so die Forscher.

Richard Kessing, Zeiskam 